\title{
The Risk of Failure: Trial and Error Learning and Long-Run Performance
}

\author{
American Economic Journal: Microeconomics
}

Steven Callander and Niko Matouschek

Online Appendix

This appendix proves the results on divergence, imitation, pilots, and longer planning horizons.

\section{Long-Run Performance}

Proof of Proposition 4: We can interpret our model as one in which, instead of a sequence of short-lived agents, there is a single, infinitely long-lived but myopic agent. For expositional convenience, we use this interpretation in this proof. We can then denote the agent in charge of Field $k=L, H$ and Agent $k$.

Suppose that Agent $L$ engages in optimal experimentation. This is going to generate some outcome $m_{1}^{L *}$ in the first period, $m_{2}^{L *}$ in the second, and so on. Now take any period $T$ and let $\tau$ denote the largest $t \in[1, T]$ in which Agent $L$ engaged in experimentation. Note that since $m_{0}^{L}>\widehat{m}$, Agent $L$ engages in experimentation in the first period and thus $\tau \in[1, T]$. We can now write the outcome Agent $L$ realized each period as

$$
m_{t}^{L *}=\left\{\begin{array}{l}
m_{t-1}^{L *}+\mu \Delta\left(m_{t-1}^{L *}\right)+\sigma \sqrt{\Delta\left(m_{t-1}^{L *}\right)} Z_{t} \text { for } t=1, \ldots, \tau \\
\bar{m}_{\tau^{L}+1}^{L *} \text { for } t=\tau+1, \ldots, T
\end{array}\right.
$$

where $Z_{t}$ is the realization of a random variable $z_{t}$ that is drawn from a standard normal distribution and where we streamline our notation by defining $m_{0}^{L *} \equiv m_{0}^{L}$.

Consider now Agent $H$. Since $m_{0}^{H}>\widehat{m}$ this agent also engages in experimentation in the first period. Suppose now that if Agent $H$ engages in experimentation in a period $t=1, \ldots, \tau$ he happens to realize the same $Z_{t}$ that Agent $L$ realized. We will show that it must then be the case that

$$
\mathrm{E}_{T+1}\left[m_{T+1}^{H *}-m_{T+1}^{L *}\right] \geq m_{0}^{H}-m_{0}^{L}
$$

where the inequality is strict for some values of $Z_{1}, \ldots, Z_{\tau}$. Since this result holds for any $T$ and any Z's, it implies (9). 
To show (26), we first need to introduce two definitions. For the first definition, consider some period $t$ in which both agents find it optimal to engage in experimentation. We know from above that each agent's outcome is given by

$$
m_{t}^{k *}=m_{t-1}^{k *}+\mu \Delta\left(m_{t-1}^{k *}\right)+\sigma \sqrt{\Delta\left(m_{t-1}^{k *}\right)} Z_{t} \quad \text { for } k=H, L
$$

We then define

$$
\widehat{z}_{t}\left(m_{t-1}^{H *}, m_{t-1}^{L *}\right) \equiv-\frac{\mu\left(\Delta\left(m_{t-1}^{H *}\right)-\Delta\left(m_{t-1}^{L *}\right)\right)}{\sigma\left(\sqrt{\Delta\left(m_{t-1}^{H *}\right)}-\sqrt{\Delta\left(m_{t-1}^{L *}\right)}\right)}
$$

as the value of $Z_{t}$ such that $m_{t}^{H *}-m_{t}^{L *}=m_{t-1}^{H *}-m_{t-1}^{L *}$. For the second definition, recall that an agent engages in experimentation in period $t+1$ if and only if $m_{t}^{*}>\widetilde{m}\left(\bar{m}_{t}\right)$, where $\bar{m}_{t}=$ $\max \left[m_{0}, m_{1}^{*}, \ldots m_{t-1}^{*}\right]$. We then define

$$
\widetilde{z}_{t}\left(m_{t-1}^{L *}, \bar{m}_{t}^{L}\right) \equiv-\frac{m_{t-1}^{L *}+\mu \Delta\left(m_{t-1}^{L *}\right)-\widetilde{m}\left(\bar{m}_{t}^{L}\right)}{\sigma \sqrt{\Delta\left(m_{t-1}^{L *}\right)}}
$$

as the value of $Z_{t}$ such that $m_{t}^{*}=m_{t-1}^{L *}+\mu \Delta\left(m_{t-1}^{L *}\right)+\sigma \sqrt{\Delta\left(m_{t-1}^{L *}\right)} Z_{t}=\widetilde{m}\left(\bar{m}_{t}^{L}\right)$.

Since Agent $L$ engages in experimentation in periods $2, \ldots, \tau$ it must be that

$$
Z_{t}>\widetilde{z}_{t}\left(m_{t-1}^{L *}, \bar{m}_{t}^{L}\right) \text { for all } t=1, \ldots, \tau-1 .
$$

In Lemma A2 below we show that if (29) holds then it must be that

$$
\widetilde{z}_{t}\left(m_{t-1}^{L *}, \bar{m}_{t}^{L}\right)>\max \left[\widetilde{z}_{t}\left(m_{t-1}^{H *}, \bar{m}_{t}^{H}\right), \widehat{z}_{t}\left(m_{t-1}^{H *}, m_{t-1}^{L *}\right)\right] \text { for all } t=1, \ldots, \tau \text {. }
$$

To see the implications of this result, suppose first that $\tau=T$, in which case Agent $L$ is experimenting in all periods up to and including period $T$. Since $Z_{t}>\widetilde{z}_{t}\left(m_{t-1}^{L *}, \bar{m}_{t}^{L}\right)$ for all $t=1, \ldots, T-1$, it follows from (30) that

$$
Z_{t}>\widetilde{z}_{t}\left(m_{t-1}^{H *}, \bar{m}_{t}^{H}\right) \quad \text { and } \quad Z_{t}>\widehat{z}_{t}\left(m_{t-1}^{H *}, m_{t-1}^{L *}\right) \text { for all } t=1, \ldots, T-1
$$

Together with the fact that $m_{0}^{H}>\widehat{m}$, the first inequality implies that Agent $H$ also engages in experimentation in all periods up to and including period $T$. And the second inequality implies that

$$
m_{T-1}^{H *}-m_{T-1}^{L *}>m_{T-2}^{H *}-m_{T-2}^{L *}>\ldots>m_{0}^{H}-m_{0}^{L} .
$$

Finally, since (30) holds for $t=T$ it must be that either (i.) $Z_{T}>\widetilde{z}_{T}\left(m_{T}^{L *}, \bar{m}_{T}^{L}\right)$, (ii.) $Z_{T}<$ $\widetilde{z}_{T}\left(m_{T}^{H *}, \bar{m}_{T}^{H}\right)$, or (iii.) $Z_{T} \in\left(\widetilde{z}_{T}\left(m_{T}^{H *}, \bar{m}_{T}^{H}\right), \widetilde{z}_{T}\left(m_{T}^{L *}, \bar{m}_{T}^{L}\right)\right)$. We will show next that (26) holds in any one of those three cases. 
Case (i.): If $Z_{T}>\widetilde{z}_{T}\left(m_{T}^{L *}, \bar{m}_{T}^{L}\right)$ both agents experiment in period $T+1$. We then have

$$
\begin{aligned}
\mathrm{E}_{T+1}\left[m_{T+1}^{H *}-m_{T+1}^{L *}\right] & =m_{T}^{H *}+\mu \Delta\left(m_{T}^{H *}\right)-m_{T}^{L *}-\mu \Delta\left(m_{T}^{L *}\right) \\
& >m_{T}^{H *}-m_{T}^{L *} \\
& >m_{0}^{H}-m_{0}^{L}
\end{aligned}
$$

where the first inequality follows from the fact that the optimal experiment is strictly increasing in the outcome. To derive the second inequality, notice that since $Z_{T}>\widehat{z}_{T}\left(m_{T-1}^{H *}, m_{T-1}^{L *}\right)$ we have $m_{T}^{H *}-m_{T}^{L *}>m_{T-1}^{H *}-m_{T-1}^{L *}$. The second inequality then follows from (29).

Case (ii.): If $Z_{T}<\widetilde{z}_{T}\left(m_{T}^{H *}, \bar{m}_{T}^{H}\right)$ neither agent experiments in period $T$. We then have

$$
\mathrm{E}_{T+1}\left[m_{T+1}^{H *}-m_{T+1}^{L *}\right]=\bar{m}_{T}^{H}-\bar{m}_{T}^{L}>m_{0}^{H}-m_{0}^{L} .
$$

To see the inequality, let $\bar{\tau}^{k} \in\{0,1, \ldots, T-1\}$ denote the period in which the outcome of Agent $k=H, L$ peaked, that is, in which $m_{\bar{\tau}^{k}}^{k *}=\bar{m}_{T}^{k *}$ (where $\bar{\tau}^{k}=0$ is the case in which the outcome was below status quo outcome in $t=1,2, \ldots, T-1)$. Suppose first that $\bar{\tau}^{H}=\bar{\tau}^{L}$. Then

$$
\bar{m}_{T}^{H}-\bar{m}_{T}^{L}=m_{\bar{\tau}^{H}}^{H *}-\bar{m}_{\bar{\tau}^{H}}^{L *}=m_{0}^{H}-m_{0}^{L} \text { if } \bar{\tau}^{H}=0 .
$$

and

$$
\bar{m}_{T}^{H}-\bar{m}_{T}^{L}=m_{\bar{\tau}^{H}}^{H *}-\bar{m}_{\bar{\tau}^{H}}^{L *}>m_{0}^{H}-m_{0}^{L} \text { if } \bar{\tau}^{H}>0
$$

where the inequality follows from (31). Suppose next that $\bar{\tau}^{H} \neq \bar{\tau}^{L}$. Then

$$
\bar{m}_{T}^{H}-\bar{m}_{T}^{L}>m_{\bar{\tau}^{L}}^{H *}-\bar{m}_{\bar{\tau}^{L}}^{L *}>m_{0}^{H}-m_{0}^{L},
$$

where the first inequality follows from $\bar{m}_{T}^{H}>m_{\bar{\tau}^{L}}^{H *}$ and $\bar{m}_{T}^{L}=m_{\bar{\tau}^{L}}^{L *}$ and the second inequality follows from (31).

Case (iii.): If $Z_{T} \in\left(\widetilde{z}_{T}\left(m_{T}^{H *}, \bar{m}_{T}^{H}\right), \widetilde{z}_{T}\left(m_{T}^{L *}, \bar{m}_{T}^{L}\right)\right)$ Agent $H$ engages in experimentation in period $T+1$ but Agent $L$ does not. Since Agent $H$ prefers engaging in experimentation to realizing his previous peak $\bar{m}_{T}^{H}$ it must be that $\mathrm{E}_{T+1}\left[m_{T+1}^{H *}\right]>\bar{m}_{T}^{H}$. We therefore have

$$
\mathrm{E}_{T+1}\left[m_{T+1}^{H *}-m_{T+1}^{L *}\right]>\bar{m}_{T}^{H}-\bar{m}_{T}^{L} \geq m_{0}^{H}-m_{0}^{L}
$$

where the second inequality follows from our discussion in Case (ii.) above.

To complete the proof, suppose that $\tau<T$. Since $\tau$ is the last period in which Agent $L$ engaged in experimentation, we have $m_{t}^{L *}=\bar{m}_{\tau}^{L}$ for all $t \geq \tau+1$. Since $Z_{t}>\widetilde{z}_{t}\left(m_{t-1}^{L *}, \bar{m}_{t}^{L}\right)$ for all $t=1, \ldots, \tau-1$, it follows from $(29)$ that

$$
Z_{t}>\widetilde{z}_{t}\left(m_{t-1}^{H *}, \bar{m}_{t}^{H}\right) \quad \text { and } \quad Z_{t}>\widehat{z}_{t}\left(m_{t-1}^{H *}, m_{t-1}^{L *}\right) \text { for all } t=1, \ldots, \tau-1
$$


Together with the fact that $m_{0}^{H}>\widehat{m}$, the first inequality implies that Agent $H$ also engages in experimentation in all periods up to and including period $\tau$. And the second inequality implies that

$$
m_{\tau-1}^{H *}-m_{\tau-1}^{L *}>m_{\tau-2}^{H *}-m_{\tau-2}^{L *}>\ldots>m_{0}^{H}-m_{0}^{L} .
$$

Finally, since (30) holds for $t=\tau$ it must be that either (a.) $Z_{\tau}<\widetilde{z}_{\tau}\left(m_{\tau}^{H *}, \bar{m}_{\tau}^{H}\right)$ or (b.) $Z_{\tau} \in$ $\left(\widetilde{z}_{\tau}\left(m_{\tau}^{H *}, \bar{m}_{\tau}^{H}\right), \widetilde{z}_{\tau}\left(m_{\tau}^{L *}, \bar{m}_{\tau}^{L}\right)\right)$. We will show next that $(26)$ holds in either of those cases:

Case (a.): If $Z_{\tau}<\widetilde{z}_{\tau}\left(m_{\tau}^{H *}, \bar{m}_{\tau}^{H}\right)$ then Agent $H$ also does not experiment in periods $t=$ $\tau+1, \ldots, T$. We then have

$$
\mathrm{E}_{T+1}\left[m_{T+1}^{H *}-m_{T+1}^{L *}\right]=\bar{m}_{\tau}^{H}-\bar{m}_{\tau}^{L} \geq m_{0}^{H}-m_{0}^{L},
$$

where the inequality follows from our discussion in Case (ii.) above.

Case (b.): If $Z_{\tau} \in\left(\widetilde{z}_{\tau}\left(m_{\tau}^{H *}, \bar{m}_{\tau}^{H}\right), \widetilde{z}_{\tau}\left(m_{\tau}^{L *}, \bar{m}_{\tau}^{L}\right)\right)$ then Agent $H$ does engage in experimentation in period $\tau+1$ and, possibly, in period $T+1$. Since, in period $T+1$, Agent $H$ can guarantee himself $\bar{m}_{\tau}^{H}$ it must be that $\mathrm{E}_{T+1}\left[m_{T+1}^{H *}\right] \geq \bar{m}_{\tau}^{H}$. We therefore have

$$
\mathrm{E}_{T+1}\left[m_{T+1}^{H *}-m_{T+1}^{L *}\right] \geq \bar{m}_{\tau}^{H}-\bar{m}_{\tau}^{L} \geq m_{0}^{H}-m_{0}^{L},
$$

where, once again, the second inequality follows from our discussion in Case (ii.) above.

LEMMA A2. If

$$
Z_{t}>\widetilde{z}_{t}\left(m_{t-1}^{L *}, \bar{m}_{t}^{L}\right) \text { for all } t=1, \ldots, \tau-1 \text {. }
$$

then

$$
\widetilde{z}_{t}\left(m_{t-1}^{L *}, \bar{m}_{t}^{L}\right)>\max \left[\widetilde{z}_{t}\left(m_{t-1}^{H *}, \bar{m}_{t}^{H}\right), \widehat{z}_{t}\left(m_{t-1}^{H *}, m_{t-1}^{L *}\right)\right] \text { for all } t=1, \ldots, \tau \text {. }
$$

Proof of Lemma A2: To prove this lemma, we first show that (35) holds for $t=1$. We then show that if (35) holds for $1, \ldots, x$, where $1<x \leq \tau-1$, then it also holds for $t=x+1$. Together these facts imply that (35) holds for $t=1, \ldots, \tau$ as claimed in the lemma.

Suppose first then that $t=1$. From the definitions of $\widehat{z}_{t}(\cdot)$ and $\widetilde{z}_{t}(\cdot)$ in $(27)$ and $(28)$ we have

$$
\widetilde{z}_{1}\left(m_{0}^{L}, m_{0}^{L}\right)-\widehat{z}_{1}\left(m_{0}^{H}, m_{0}^{L}\right)=\frac{\widetilde{m}\left(m_{0}^{L}\right)+\mu \sqrt{\Delta\left(m_{0}^{L}\right)} \sqrt{\Delta\left(m_{0}^{H}\right)}-m_{0}^{L}}{\sigma \sqrt{\Delta\left(m_{0}^{L}\right)}} .
$$


and

$$
\begin{aligned}
& \widetilde{z}_{1}\left(m_{0}^{L}, m_{0}^{L}\right)-\widetilde{z}_{1}\left(m_{0}^{H}, m_{0}^{H}\right)=\frac{m_{0}^{H}-m_{0}^{L}+\widetilde{m}\left(m_{0}^{L}\right)-\widetilde{m}\left(m_{0}^{H}\right)}{\sigma \sqrt{\Delta\left(m_{0}^{H}\right)}} \\
& +\frac{\sqrt{\Delta\left(m_{0}^{H}\right)}-\sqrt{\Delta\left(m_{0}^{L}\right)}}{\sigma \sqrt{\Delta\left(m_{0}^{L}\right)} \sqrt{\Delta\left(m_{0}^{H}\right)}}\left(\widetilde{m}\left(m_{0}^{L}\right)+\mu \sqrt{\Delta\left(m_{0}^{L}\right)} \sqrt{\Delta\left(m_{0}^{H}\right)}-m_{0}^{L}\right) .
\end{aligned}
$$

To see that (36) is strictly positive notice that

$$
\begin{aligned}
& \tilde{m}\left(m_{0}^{L}\right)+\mu \sqrt{\Delta\left(m_{0}^{L}\right)} \sqrt{\Delta\left(m_{0}^{H}\right)}-m_{0}^{L} \\
> & \tilde{m}\left(m_{0}^{L}\right)+\mu \Delta\left(m_{0}^{L}\right)-m_{0}^{L} \\
> & \tilde{m}\left(m_{0}^{L}\right)+\mu \Delta\left(\widetilde{m}\left(m_{0}^{L}\right)\right)-m_{0}^{L} \\
> & 0,
\end{aligned}
$$

where the first inequality follows from $\Delta\left(m_{0}^{H}\right)>\Delta\left(m_{0}^{L}\right)$ and the second follows from $m_{0}^{L}>$ $\widetilde{m}\left(m_{0}^{L}\right)$. To see the third inequality, recall that $\widetilde{m}\left(m_{0}^{L}\right)$ is defined as the outcome at which the agent is indifferent between receiving $m_{0}^{L}$ and a normally distributed gamble that pays $\widetilde{m}\left(m_{0}^{L}\right)+$ $\mu \Delta\left(\widetilde{m}\left(m_{0}^{L}\right)\right)$ on average and has a strictly positive variance $\Delta\left(\widetilde{m}\left(m_{0}^{L}\right)\right) \sigma^{2}$. Since the agent is risk averse, it must then be that $\widetilde{m}\left(m_{0}^{L}\right)+\mu \Delta\left(\widetilde{m}\left(m_{0}^{L}\right)\right)>m_{0}^{L}$.

To show that (37) is also strictly positive, consider first the second term on the RHS of (37). Since $\Delta\left(m_{0}^{H}\right)>\Delta\left(m_{0}^{L}\right)$ it follows from (38) that this term is strictly positive. Consider next the first term on the RHS of (37). This term has to be weakly positive since $m_{0}^{H}>m_{0}^{L}$ and $\mathrm{d} \widetilde{m}(m) / \mathrm{d} m \in(0,1]$. We therefore have $\widetilde{z}_{1}\left(m_{0}^{L}, m_{0}^{L}\right)>\max \left[\widetilde{z}_{1}\left(m_{0}^{H}, m_{0}^{H}\right)>\widehat{z}_{t}\left(m_{0}^{H}, m_{0}^{L}\right)\right]$.

Suppose now that (35) holds for $t=1, \ldots, x$, where $1<x \leq \tau-1$. We will show that (35) then also holds for $t=x+1$. For this purpose, notice first that if (35) holds for $t=1, \ldots, x$, then it follows from (34) that (i.) both agents are engaging in experimentation in periods $t=1, \ldots, x+1$ and (ii.) it must be that

$$
m_{x+1}^{H *}-m_{x+1}^{L *}>m_{x}^{H *}-m_{x}^{L *}>\ldots>m_{0}^{H}-m_{0}^{L} .
$$

Furthermore, we know from the definitions of $\widehat{z}_{t}(\cdot)$ and $\widetilde{z}_{t}(\cdot)$ in $(27)$ and $(28)$ that

$$
\widetilde{z}_{x+1}\left(m_{x}^{L *}, \bar{m}_{x+1}^{L}\right)-\widehat{z}_{x+1}\left(m_{x}^{H *}, m_{x}^{L *}\right)=\frac{\widetilde{m}\left(\bar{m}_{x+1}^{L}\right)+\mu \sqrt{\Delta\left(m_{x}^{L *}\right)} \sqrt{\Delta\left(m_{x}^{H *}\right)}-m_{x}^{L *}}{\sigma \sqrt{\Delta\left(m_{x}^{L *}\right)}} .
$$


To see that this expression is strictly positive, notice that

$$
\begin{aligned}
& \widetilde{m}\left(\bar{m}_{x+1}^{L}\right)+\mu \sqrt{\Delta\left(m_{x}^{L *}\right)} \sqrt{\Delta\left(m_{x}^{H *}\right)}-m_{x}^{L *} \\
\geq & \widetilde{m}\left(m_{x}^{L}\right)+\mu \sqrt{\Delta\left(m_{x}^{L *}\right)} \sqrt{\Delta\left(m_{x}^{H *}\right)}-m_{x}^{L *} \\
> & \widetilde{m}\left(m_{x}^{L}\right)+\mu \Delta\left(m_{x}^{L *}\right)-m_{x}^{L *} \\
> & \widetilde{m}\left(m_{x}^{L}\right)+\mu \Delta\left(\widetilde{m}\left(m_{x}^{L}\right)\right)-m_{x}^{L *} \\
> & 0
\end{aligned}
$$

where the first inequality follows from $\bar{m}_{x+1}^{L} \geq m_{x}^{L}$, the second from $\Delta\left(m_{x}^{H *}\right)>\Delta\left(m_{x}^{L *}\right)$, and the third from $m_{x}^{L *}>\widetilde{m}\left(m_{x}^{L}\right)$. To derive the last inequality, recall that $\widetilde{m}\left(m_{x}^{L}\right)$ is defined as the outcome at which the agent is indifferent between receiving $m_{x}^{L}$ and a normally distributed gamble that pays $\widetilde{m}\left(m_{x}^{L}\right)+\mu \Delta\left(\widetilde{m}\left(m_{x}^{L}\right)\right)$ on average and has a strictly positive variance $\Delta\left(\widetilde{m}\left(m_{x}^{L}\right)\right) \sigma^{2}$. Since the agent is risk averse, it must then be that $\widetilde{m}\left(m_{x}^{L}\right)+\mu \Delta\left(\widetilde{m}\left(m_{x}^{L}\right)\right)-m_{x}^{L *}$. We therefore have that if (35) holds for $t=1, \ldots, x$, then $\widetilde{z}_{x+1}\left(m_{x}^{L *}, \bar{m}_{x+1}^{L}\right)>\widehat{z}_{x+1}\left(m_{x}^{H *}, m_{x}^{L *}\right)$.

Next, we know from the definition of $\widetilde{z}_{t}(\cdot)$ in $(28)$ that

$$
\begin{aligned}
& \widetilde{z}_{x+1}\left(m_{x}^{L *}, \bar{m}_{x}^{L *}\right)-\widetilde{z}_{x+1}\left(m_{x}^{H *}, \bar{m}_{x}^{H *}\right)=\frac{m_{x}^{H *}-m_{x}^{L *}+\widetilde{m}\left(\bar{m}_{x+1}^{L *}\right)-\widetilde{m}\left(\bar{m}_{x+1}^{H *}\right)}{\sigma \sqrt{\Delta\left(m_{x}^{H *}\right)}} \\
& +\frac{\sqrt{\Delta\left(m_{x}^{H *}\right)}-\sqrt{\Delta\left(m_{x}^{L *}\right)}}{\sigma \sqrt{\Delta\left(m_{x}^{L *}\right)} \sqrt{\Delta\left(m_{x}^{H *}\right)}}\left(\widetilde{m}\left(\bar{m}_{x+1}^{L *}\right)+\mu \sqrt{\Delta\left(m_{x}^{L *}\right)} \sqrt{\Delta\left(m_{x}^{H *}\right)}-m_{x}^{L *}\right) .
\end{aligned}
$$

We know from (40) that the first term on the RHS is strictly positive. To show that the second term is weakly positive, we first show that

$$
m_{x}^{H *}-m_{x}^{L *} \geq \bar{m}_{x+1}^{H *}-\bar{m}_{x+1}^{L *} .
$$

For this purpose, let $\bar{\tau}^{k} \in\{0,1, \ldots, x\}$ denote the period in which the outcome of Agent $k=H, L$ peaked, that is, in which $m_{\bar{\tau}^{k}}^{k *}=\bar{m}_{x+1}^{k *}$ (where $\bar{\tau}^{k}=0$ is the case in which the outcome was below the status quo outcome in $t=1,2, \ldots, x)$. Suppose now that $\bar{\tau}^{H}=\bar{\tau}^{L}$. Then

$$
m_{x}^{H *}-m_{x}^{L *} \geq m_{\bar{\tau}^{H}}^{H *}-m_{\bar{\tau}^{H}}^{L *}=\bar{m}_{x+1}^{H *}-\bar{m}_{x+1}^{L *},
$$

where the inequality follows from (39) and the equality follows from the definition of $\bar{\tau}^{H}$ and $\bar{\tau}^{L}$.

Suppose next that $\bar{\tau}^{H} \neq \bar{\tau}^{L}$. Then

$$
m_{x}^{H *}-m_{x}^{L *} \geq m_{\bar{\tau}^{H}}^{H *}-m_{\bar{\tau}^{H}}^{L *}>m_{\bar{\tau}^{H}}^{H *}-m_{\bar{\tau}^{L}}^{L *}=\bar{m}_{x+1}^{H *}-\bar{m}_{x+1}^{L *},
$$

where the first inequality follows from (39), the second follows from $m_{\bar{\tau}^{L}}^{L *}>m_{\bar{\tau}^{H}}^{L *}$, and the equality follows from the definition of $\bar{\tau}^{H}$ and $\bar{\tau}^{L}$. We therefore have $m_{x}^{H *}-m_{x}^{L *} \geq \bar{m}_{x+1}^{H *}-\bar{m}_{x+1}^{L *}$ as claimed above. 
Finally, notice that

$$
\bar{m}_{x+1}^{H *}-\bar{m}_{x+1}^{L *} \geq \widetilde{m}\left(\bar{m}_{x+1}^{H *}\right)-\widetilde{m}\left(\bar{m}_{x+1}^{L *}\right),
$$

where the inequality follows from $\bar{m}_{x}^{H *}>\bar{m}_{x}^{L *}$ and $\mathrm{d} \widetilde{m}(m) / \mathrm{d} m \in(0,1]$. We therefore have

$$
m_{x}^{H *}-m_{x}^{L *} \geq \bar{m}_{x+1}^{H *}-\bar{m}_{x+1}^{L *} \geq \widetilde{m}\left(\bar{m}_{x+1}^{H *}\right)-\widetilde{m}\left(\bar{m}_{x+1}^{L *}\right)
$$

which implies that the second term on the RHS of (41) is weakly positive.

Proof of Proposition 5: The logic of escape is easiest to see for the example utility function of Section 4.4. We prove the result in this special case. The key property in the argument is that the experiment step size increases without bound in $m$. As this property holds generally, it is straightforward to extend the argument to the full model.

We establish the result by proving a stronger result: That the probability agents achieve at least half of the expected gain in performance in every period is strictly bounded away from zero. Beginning at $m_{0}>\hat{m}$, this critical threshold we denote $m_{1}^{\#}$, is $m_{0}+\frac{1}{2}\left(m_{0}-\hat{m}\right) c \mu$, where $c>0$ is a constant. The probability of success is $1-C D F\left(m_{1}^{\#}\right)=\frac{1}{2}\left[1-\operatorname{erf}\left(m_{1}^{\#}\right)\right]=1-\frac{1}{2} \operatorname{erfc}\left(-m_{1}^{\#}\right)$, where erf and $\operatorname{erfc}$ are the error function and complementary error function, respectively, and using the identities erf $(x)=-\operatorname{erf}(-x)$ and $\operatorname{erfc}(x)=1-\operatorname{erf}(x)$. From Chang, Cosman, and Milstein (2011) we have $\operatorname{erfc}(x) \leq e^{-x^{2}}$, and thus the success probability satisfies:

$$
\underset{e}{\operatorname{Pr}}(1) \geq 1-\frac{1}{2} e^{-\left[\frac{\frac{1}{2}\left(m_{0}-\hat{m}\right) c \mu}{\sigma \sqrt{2\left(m_{0}-\hat{m}\right) c}}\right]^{2}}
$$

Take $m_{1}^{\#}$ as the realized outcome and iterate. The same set of calculations give:

$$
\operatorname{Pr}(2) \geq 1-\frac{1}{2} e^{-\left[\frac{\frac{1}{2}\left(m_{0}-\hat{m}\right)\left(\frac{1}{2} c \mu+1\right) c \mu}{\sigma \sqrt{2\left(m_{0}-\hat{m}\right)\left(\frac{1}{2} c \mu+1\right) c}}\right]^{2}}=1-\frac{1}{2} e^{-\left[\frac{\mu^{2}}{8 \sigma^{2}}\right]\left(m_{0}-\hat{m}\right)\left(\frac{1}{2} c \mu+1\right) c}
$$

And this generalizes to:

$$
\operatorname{Pr}_{e}(t) \geq 1-\frac{1}{2} e^{-\left[\frac{\mu^{2}}{8 \sigma^{2}}\right]\left(m_{0}-\hat{m}\right)\left(\frac{1}{2} c \mu+1\right)^{t-1} c}
$$

The escape probability is:

$$
\underset{e}{\operatorname{Pr}} \geq \prod_{t=1}^{\infty} \operatorname{Pr}(t)=\prod_{t=1}^{\infty}\left[1-\frac{1}{2} e^{-w z^{t-1}}\right]
$$

where $w>0$ and $z>1$ are constants.

$$
\begin{aligned}
& \text { As } 0<1-e^{-w z^{t-1}}<1, \\
& \qquad \prod_{t=1}^{T}\left(1-\frac{1}{2} e^{-w z^{t-1}}\right)
\end{aligned}
$$


converges as $T \rightarrow \infty$. Taking the logarithm of this product, we get that the product converges to a positive value if

$$
\sum_{t=1}^{T} \ln \left(1-\frac{1}{2} e^{-w z^{t-1}}\right)
$$

converges as $T \rightarrow \infty$. To prove that, we first note the classical inequality ${ }^{11}$

$$
\ln (1-x) \geq-\frac{3}{2} x
$$

for $0 \leq x \leq 1 / 2$. We also note that since $z>1, z^{t} / t \rightarrow \infty$ and so there exists $T_{0}$ such that if $t \geq T_{0}, z^{t-1}>t$. Hence, for any $T>T_{0}$, we have the inequality

$$
\sum_{t=T_{0}}^{T} \ln \left(1-\frac{1}{2} e^{-w z^{t-1}}\right) \geq \sum_{t=T_{0}}^{T} \ln \left(1-\frac{1}{2} e^{-w t}\right) \geq-\frac{3}{2} \sum_{t=T_{0}}^{T} \frac{1}{2} e^{-w t} \geq-\frac{3}{4} \sum_{t=0}^{\infty} e^{-w t}=-\frac{3}{4} \cdot \frac{1}{1-e^{-w}}
$$

as each term $\ln \left(1-e^{-w z^{t-1}}\right)$ is negative. This establishes the necessary convergence.

\section{Longer Planning Horizons}

We assume that $\beta>2 \frac{\mu}{\sigma^{2}}$, which ensures that the crossing condition is satisfied, and that $m_{0}>\widehat{m}$, which ensures that the first agent engages in experimentation.

PROPOSITION 6. In the second period of their lives, agents behave as in the main model. An optimal action therefore exists and is given by

$$
a_{t}^{*}= \begin{cases}\bar{a}_{t} & \text { if } m\left(h_{t}\right) \leq \widetilde{m}\left(m\left(h_{t}\right)\right) \\ h_{t}+\Delta\left(m\left(h_{t}\right)\right) & \text { if } m\left(h_{t}\right) \geq \widetilde{m}\left(m\left(h_{t}\right)\right),\end{cases}
$$

where

$$
\begin{gathered}
\Delta\left(m\left(h_{t}\right)\right)=\max \left\{0,2 \frac{m\left(h_{t}\right)-\widehat{m}}{\sigma^{2}\left(\beta-\frac{2 \mu}{\sigma^{2}}\right)}\right\}, \\
\widehat{m}=-\frac{1}{\beta} \ln \left[\frac{2 \alpha \mu}{\sigma^{2} \beta\left(\beta-2 \frac{\mu}{\sigma^{2}}\right)}\right],
\end{gathered}
$$

and

$$
\widetilde{m}\left(m\left(h_{t}\right)\right)=\widehat{m}+\frac{\beta-\frac{2 \mu}{\sigma^{2}}}{\alpha \beta}\left(u\left(m\left(\bar{a}_{t}\right)\right)-u(\widehat{m})\right) .
$$

The agent's expected utility from taking the best action is given by

$W\left(m\left(h_{t}\right)+\mu\left(a_{t}^{*}-h_{t}\right),\left(a_{t}^{*}-h_{t}\right) \sigma^{2}\right)= \begin{cases}u\left(m\left(\bar{a}_{t}\right)\right) & \text { if } m\left(h_{t}\right) \leq \widetilde{m}\left(m\left(h_{t}\right)\right) \\ u(\widehat{m})+\left(m\left(h_{t}\right)-\widehat{m}\right) \frac{\alpha \beta}{\left(\beta-\frac{2 \mu}{\sigma^{2}}\right)} & \text { if } m\left(h_{t}\right) \geq \widetilde{m}\left(m\left(h_{t}\right)\right) .\end{cases}$

\footnotetext{
${ }^{11}$ For example, see http://functions.wolfram.com/ElementaryFunctions/Log/29/
} 
Proof: In the second period of their lives, the agents only care about expected utility from that period. They therefore behave just like agents in the main model. Given the exponential utility function, expected utility from taking action $h_{t}+\Delta_{t}$ is given by

$$
W\left(m\left(h_{t}\right)+\mu \Delta_{t}, \Delta_{t} \sigma^{2}\right)=\alpha\left(m\left(h_{t}\right)+\mu \Delta_{t}\right)-\exp \left(-\beta\left(m\left(h_{t}\right)+\mu \Delta_{t}\right)+\frac{1}{2} \beta^{2} \Delta_{t} \sigma^{2}\right) .
$$

The expressions in the proposition then follow from Propositions 1-3.

PROPOSITION 8. In period $t=1$ an optimal action exists. If, as we assume, $m_{0}>\widehat{m}$, any optimal action is strictly to the right of $a_{0}$ and increasing in the discount factor $\delta$, where $\widehat{m}$ is defined in (43). If, instead, $m_{0}$ were weakly smaller than $\widehat{m}$, the agent would take the status quo action $a_{0}$.

Proof: In the first period it can never be optimal for the agent to take an action strictly to the left of $a_{0}$. If it exists, the optimal first period action is therefore weakly to the right of $a_{0}$. The problem of characterizing the optimal first period actions that are weakly to the right of $a_{0}$ is a special case of the problem of characterizing the optimal actions in any period $t$ in which a agent is in the first period of his life and is constrained to taking an action weakly to the right of the right-most action $h_{t}$. Since this more general problem is relevant for the proof of the next proposition, we examine it here.

Consider then any period $t$ in which a agent is in the first period of his life and suppose that he has to take an action $a_{t} \geq h_{t}$. We know from the previous proposition that in $t+1$ the agent will then experiment to the right of $a_{t}$ if and only if

$$
m\left(a_{t}\right) \geq \widetilde{m}\left(\bar{m}_{t+1}\right)
$$

This optimal learning rule is equivalent to the agent experimenting to the right if and only if

$$
m\left(a_{t}\right) \geq \widetilde{m}\left(\bar{m}_{t}\right)
$$

To see this, notice that the two inequalities are only different if

$$
m\left(a_{t}\right)>\max \left\{m_{0}, \ldots, m_{t-1}\right\} .
$$

Since

$$
m\left(a_{t}\right) \geq \widetilde{m}\left(m\left(a_{t}\right)\right)
$$

and

$$
\max \left\{m_{0}, \ldots, m_{t-1}\right\}>\widetilde{m}\left(\max \left\{m_{0}, \ldots, m_{t-1}\right\}\right)
$$


inequality (48) implies (46) and (47) which, in turn, implies that the two learning rules are equivalent.

Next, we can write $m\left(a_{t}\right)$ as

$$
m\left(a_{t}\right)=m\left(h_{t}\right)+\mu \Delta_{t}+\sigma \sqrt{\Delta_{t}} z_{t} .
$$

Substituting this expression into (47) and rearranging, we have that in period $t+1$ the agent experiments to the right if and only if

$$
z_{t} \geq \widetilde{z}_{t}
$$

where

$$
m\left(h_{t}\right)+\mu \Delta_{t}+\sigma \sqrt{\Delta_{t}} \widetilde{z}=\widetilde{m}\left(\bar{m}_{t}\right)
$$

or equivalently

$$
\widetilde{z}_{t}=\left(-\frac{m\left(h_{t}\right)-\widetilde{m}\left(\bar{m}_{t}\right)+\mu \Delta_{t}}{\sigma \sqrt{\Delta_{t}}}\right) .
$$

We can therefore write the agent's problem as

$$
\max _{\Delta_{t} \geq 0} V_{r}
$$

where

$$
\begin{aligned}
& V_{r}= \alpha\left(m\left(h_{t}\right)+\mu \Delta_{t}\right)-\exp \left(-\beta\left(m\left(h_{t}\right)+\mu \Delta_{t}\right)+\frac{1}{2} \beta^{2} \Delta_{t} \sigma^{2}\right) \\
&+\delta\left[\int_{-\infty}^{\widetilde{z}} u\left(\bar{m}_{t}\right) \mathrm{d} F\left(z_{t}\right)+\int_{\widetilde{z}}^{\infty} u(\widehat{m})+\left(m\left(h_{t}\right)+\mu \Delta_{t}+\sigma \sqrt{\Delta_{t}} z_{t}-\widehat{m}\right) \frac{\alpha \beta}{\left(\beta-\frac{2 \mu}{\sigma^{2}}\right)} \mathrm{d} F\left(z_{t}\right)\right]
\end{aligned}
$$

and the subscript ' $r$ ' stands for 'to the right of $h_{t}$.' Differentiating $V_{r}$ we get

$$
\begin{aligned}
\frac{\mathrm{d} V_{r}}{\mathrm{~d} \Delta_{t}}= & \alpha \mu-\frac{1}{2} \beta \sigma^{2}\left(\beta-\frac{2 \mu}{\sigma^{2}}\right) \exp \left(-\beta\left(m\left(h_{t}\right)+\mu \Delta_{t}\right)+\frac{1}{2} \beta^{2} \Delta_{t} \sigma^{2}\right) \\
& +\delta \frac{\alpha \beta}{\left(\beta-\frac{2 \mu}{\sigma^{2}}\right)}\left(\mu\left(1-F\left(\widetilde{z}_{t}\right)\right)+\sigma \frac{1}{2 \sqrt{\Delta_{t}}} f\left(\widetilde{z}_{t}\right)\right) .
\end{aligned}
$$

Taking limits we further have that

$$
\lim _{\Delta_{t} \rightarrow 0} \frac{\mathrm{d} V_{r}}{\mathrm{~d} \Delta_{t}}= \begin{cases}>0 & \text { if } m\left(h_{t}\right)>\widehat{m} \\ =0 & \text { if } m\left(h_{t}\right)=\widehat{m} \\ <0 & \text { if } m\left(h_{t}\right)<\widehat{m}\end{cases}
$$

and

$$
\lim _{\Delta_{t} \rightarrow \infty} \frac{\mathrm{d} V_{r}}{\mathrm{~d} \Delta_{t}}=-\infty
$$


This implies that if $m\left(h_{t}\right)>\widehat{m}$ there exists an optimal $\Delta>0$ that maximizes the agent's expected utility. Moreover, since

$$
\frac{\mathrm{d}^{2} V_{r}}{\mathrm{~d} \Delta_{t} \mathrm{~d} \delta}=\frac{\alpha \beta}{\left(\beta-\frac{2 \mu}{\sigma^{2}}\right)}\left(\mu\left(1-F\left(\widetilde{z}_{t}\right)\right)+\sigma \frac{1}{2 \sqrt{\Delta_{t}}} f\left(\widetilde{z}_{t}\right)\right) \geq 0
$$

the optimal action is increasing in $\delta$.

To establish the agent's optimal action if $m\left(h_{t}\right) \leq \widehat{m}$ notice that

$$
\begin{aligned}
\frac{\mathrm{d}^{2} V_{r}}{\mathrm{~d} \Delta_{t} \mathrm{~d} m\left(h_{t}\right)}= & \frac{1}{2} \beta^{2} \sigma^{2}\left(\beta-\frac{2 \mu}{\sigma^{2}}\right) \exp \left(-\beta\left(m\left(h_{t}\right)+\mu \Delta_{t}\right)+\frac{1}{2} \beta^{2} \Delta_{t} \sigma^{2}\right) \\
& +\delta \frac{\alpha \beta}{\left(\beta-\frac{2 \mu}{\sigma^{2}}\right)}\left(\mu \Delta_{t}+\widetilde{m}\left(\bar{m}_{t}\right)-m\left(h_{t}\right)\right) \frac{1}{\sigma 2 \sqrt{\Delta_{t}} \Delta_{t}} f\left(\widetilde{z}_{t}\right)
\end{aligned}
$$

which is strictly positive for all $\Delta_{t} \geq 0$ if $m\left(h_{t}\right) \leq \widetilde{m}\left(\bar{m}_{t}\right)$. Since $\widehat{m}<\widetilde{m}\left(\bar{m}_{t}\right)$ it then follows from (51) that if $m\left(h_{t}\right) \leq \widehat{m}$ the agent's optimal action to the right of $h_{t}$ is given by $h_{t}$.

PROPOSITION 9. Consider any period $t=3,5, \ldots$ in which one of the agents that follows the first agent is in the first period of his life. In any such period, an optimal action exists. Any optimal action is strictly to the right of $h_{t}$ if $m\left(h_{t}\right) \geq m_{h, t}$ and it is an unknown action to the left of $h_{t}$ if $m\left(h_{t}\right) \leq m_{l, t}$, where $m_{l, t}$ and $m_{h, t} \geq m_{l}$ are defined in the proof. Moreover, there exists a $\bar{\delta}>0$ such that $m_{l, t}=m_{h, t}$ for all $\delta \leq \bar{\delta}$.

Proof: In the proof of the previous proposition we characterized the solution to the problem that an agent in the first period of his life faces if he has to take an action to the right of $h_{t}$. Consider now an alternative constrained problem in which the agent has to take an action between two neighboring actions $a_{l}$ and $a_{h}$ with $a_{l}<a_{r} \leq h_{t}$ and $m\left(a_{l}\right) \geq m\left(a_{h}\right)$. The agent's expected utility is then given by

$$
\begin{aligned}
V_{l}= & \mathrm{E}\left[u\left(\mathrm{E}\left[m\left(a_{t}\right)\right]+\sqrt{\operatorname{Var}\left(m\left(a_{t}\right)\right)} z_{t}\right)\right]+\delta \int_{\widetilde{z}_{t}}^{\infty} u\left(\mathrm{E}\left[m\left(a_{t}\right)\right]+\sqrt{\operatorname{Var}\left(m\left(a_{t}\right)\right)} z_{t}\right) \mathrm{d} F\left(z_{t}\right)(52) \\
& +\delta \int_{-\infty}^{\widetilde{z}_{t}} \max \left\{u\left(m\left(\bar{a}_{t}\right)\right), \mathrm{E}\left[u\left(m\left(h_{t}\right)+\mu \Delta\left(h_{t}\right)+\sigma \sqrt{\Delta\left(h_{t}\right)} z_{t+1}\right)\right]\right\} \mathrm{d} F\left(z_{t}\right),
\end{aligned}
$$

where

$$
\begin{gathered}
\mathrm{E}\left[m\left(a_{t}\right)\right]=\frac{a_{t}-a_{l}}{a_{r}-a_{l}} m\left(a_{r}\right)+\frac{a_{r}-a_{t}}{a_{r}-a_{l}} m\left(a_{l}\right), \\
\operatorname{Var}\left(m\left(a_{t}\right)\right)=\frac{\left(a_{t}-a_{l}\right)\left(a_{r}-a_{t}\right)}{a_{r}-a_{l}} \sigma^{2},
\end{gathered}
$$




$$
\begin{gathered}
\mathrm{E}\left[m\left(a_{t}\right)\right]+\sqrt{\operatorname{Var}\left(m\left(a_{t}\right)\right)} \widetilde{z}_{t}=\overline{\bar{m}}, \\
u(\overline{\bar{m}})=\max \left\{u\left(m\left(\bar{a}_{t}\right)\right), \mathrm{E}\left[u\left(m\left(h_{t}\right)+\mu \Delta\left(h_{t}\right)+\sigma \sqrt{\Delta\left(h_{t}\right)} z_{t+1}\right)\right]\right\},
\end{gathered}
$$

and where the subscript ' $l$ ' stands for 'to the left of $h_{t}$.' Differentiating $V_{l}$ and taking limits we get

$$
\lim _{a_{t} \rightarrow a_{l}} \frac{\mathrm{d} V_{l}}{\mathrm{~d} a_{t}}=\left\{\begin{array}{cc}
\infty & \text { if } m\left(a_{l}\right)=\overline{\bar{m}} \\
\frac{1}{2} \sigma^{2} u^{\prime}\left(a_{l}\right)\left(-\frac{2}{\sigma^{2}} \frac{m\left(a_{l}\right)-m\left(a_{r}\right)}{\left(a_{r}-a_{l}\right)}-r\left(m\left(a_{l}\right)\right)\right)<0 & \text { if } m\left(a_{l}\right)<\overline{\bar{m}}
\end{array}\right.
$$

where $r(\cdot)$ is the coefficient of absolute risk aversion. If $m\left(a_{l}\right)=\overline{\bar{m}}$, therefore, there exists an action strictly between $a_{l}$ and $a_{r}$ that the agent strictly prefers to both $a_{l}$ and $a_{r}$.

Suppose now that $m\left(h_{t}\right) \leq \widehat{m}$. We have established in the proof of the previous proposition that a young agent then prefers $h_{t}$ to any action to the right of $h_{t}$. Moreover, if $m\left(h_{t}\right) \leq \widehat{m}$, it must be that $m\left(\bar{a}_{t}\right)=\overline{\bar{m}}$, in which case there exists an unknown action between $\bar{a}_{t}$ and its neighboring actions that the agent prefers to $\bar{a}_{t}$ and thus all all other known actions. For any $m\left(h_{t}\right) \leq m_{l, t} \equiv \widehat{m}$, it is therefore optimal for the agent to take an unknown action to the left of $h_{t}$.

Next, it is immediate that if $h_{t}$ is the best known action, there always exists an action to the right of $h_{t}$ that the agent prefers to any action to the left of $h_{t}$. For any $m\left(h_{t}\right) \geq m_{h, t} \equiv \bar{m}_{t-1}$, it is therefore optimal for the agent to take an unknown action to the right of $h_{t}$.

Suppose next that $m\left(h_{t}\right) \in\left(\widehat{m}, \widetilde{m}\left(\bar{m}_{t-1}\right)\right)$. The agent's expected utility from taking the best action to the left of $h_{t}$ is bounded from below by $u\left(m\left(\bar{a}_{t}\right)\right)$. Suppose now that $\delta=0$. The agent's optimal action to the right of $h_{t}$ is then given by $\Delta\left(m\left(h_{t}\right)\right)$ and his expected utility from taking this action is given by $W\left(m\left(h_{t}\right)+\mu \Delta\left(m\left(h_{t}\right)\right), \Delta\left(m\left(h_{t}\right)\right) \sigma^{2}\right)<u\left(m\left(\bar{a}_{t}\right)\right)$, where $\Delta\left(m\left(h_{t}\right)\right)$ and $W(\cdot, \cdot)$ are given by (42) and (45). Moreover, it follows from (50) that at $\delta=0$, the derivative of the agent's expected utility from experimenting to the right of $h_{t}$ is given by

$\frac{\mathrm{d} V_{r}}{\mathrm{~d} \delta}=\int_{-\infty}^{\widetilde{z}} u\left(\bar{m}_{t}\right) \mathrm{d} F\left(z_{t}\right)+\int_{\widetilde{z}}^{\infty} u(\widehat{m})+\left(m\left(h_{t}\right)+\mu \Delta\left(m\left(h_{t}\right)\right)+\sigma \sqrt{\Delta\left(m\left(h_{t}\right)\right)} z_{t}-\widehat{m}\right) \frac{\alpha \beta}{\left(\beta-\frac{2 \mu}{\sigma^{2}}\right)} \mathrm{d} F\left(z_{t}\right)$,

where $\widetilde{z}$ is defined in (49). Since this derivative is finite, it follows that there exists a $\bar{\delta}>0$ such that for all $\delta \leq \bar{\delta}$ the expected utility from taking the best action to the left of $h_{t}$ is strictly larger than the expected utility from taking the best action to the right of $h_{t}$.

Finally, suppose that $m\left(h_{t}\right) \in\left(\widetilde{m}\left(\bar{m}_{t-1}\right), \bar{m}_{t-1}\right)$, in which case $m\left(\bar{a}_{t}\right)<\overline{\bar{m}}$. As we observed above, there then exists a $\bar{\delta}>0$ such that for all $\delta \leq \bar{\delta}$ the agent never finds it optimal to take an action strictly in between two known actions. For any such $\delta$, the best action to the left of $h_{t}$ is therefore given by $\bar{a}_{t}$. The expected utility from taking the best action to the right of $h_{t}$ is bounded from below by $W\left(m\left(h_{t}\right)+\mu \Delta\left(m\left(h_{t}\right)\right), \Delta\left(m\left(h_{t}\right)\right) \sigma^{2}\right)$, where $\Delta\left(m\left(h_{t}\right)\right)$ 
is the best myopic action to the right of $h_{t}$. Since $m\left(h_{t}\right) \in\left(\widetilde{m}\left(\bar{m}_{t-1}\right), \bar{m}_{t-1}\right)$, we know that $W\left(m\left(h_{t}\right)+\mu \Delta\left(m\left(h_{t}\right)\right), \Delta\left(m\left(h_{t}\right)\right) \sigma^{2}\right)>u\left(m\left(\bar{a}_{t}\right)\right)$, which implies that for all $\delta \leq \bar{\delta}$ the expected utility from experimenting to the right of $h_{t}$ is strictly larger than that from experimenting to the left of $h_{t}$.

There therefore exists a $\bar{\delta}>0$ such that for all $\delta \leq \bar{\delta}, m_{l, t}=m_{h, t}=\widetilde{m}\left(\bar{m}_{t-1}\right)$.

\section{Pilots}

PROPOSITION 7. In any period $t \geq 1$ there exists a unique, optimal action for the agent. If $m\left(h_{t}\right) \leq \widetilde{m}\left(\bar{m}_{t}\right)$, the agent puts all his income into the best known action $\bar{a}_{t}$, where $\widetilde{m}\left(\bar{m}_{t}\right)$ is defined in the proof. If $m\left(h_{t}\right)>\widetilde{m}\left(\bar{m}_{t}\right)$, the agent puts a fraction $(1-\underline{\theta})$ of his income into the best known action $\bar{a}_{t}$ and the rest into action $h_{t}+\Delta\left(m\left(h_{t}\right)\right)$, where $\Delta\left(m\left(h_{t}\right)\right)>0$ is defined in the proof. The optimal step size $\Delta\left(m\left(h_{t}\right)\right)$ is increasing in $\mu$ and $\bar{m}_{t}$ and decreasing in $\sigma^{2}$ and the agent's risk aversion. The threshold $\widetilde{m}\left(\bar{m}_{t}\right)$ is decreasing in $\mu$, increasing $\sigma^{2}$ and the agent's risk aversion, and can be increasing or decreasing in $\bar{m}_{t}$. Moreover, an increase in the minimum feasible scale of a pilot $\underline{\theta}$ leads to an increase in the $\widetilde{m}\left(\bar{m}_{t}\right)$ and a reduction in $\Delta\left(m\left(h_{t}\right)\right)$.

Proof: Notice first that the agent will never take a known action other than the best known action, that he will never take an unknown action to the left of the right-most action $h_{t}$, and that the agent will also never take two unknown action to the left of $h_{t}$. The first two claims are immediate. To prove the last claim, suppose that in some period $t$ the agent puts $\left(1-\theta_{t}\right)$ of his income into an action $a^{\prime}>h_{t}$ and the rest into another action $a>a^{\prime}$, where $\theta_{t} \in(0,1)$. Let $\Delta^{\prime}=a^{\prime}-h_{t}$ and $\Delta=a-a^{\prime}$. The agent's expected outcome and its variance are then given by

$$
\mathrm{E}\left[m_{t}\right]=m\left(h_{t}\right)+\mu\left(\Delta^{\prime}+\theta_{t} \Delta\right)
$$

and

$$
\operatorname{Var}\left(m_{t}\right)=\sigma^{2}\left(\Delta^{\prime}+\theta_{t}^{2} \Delta\right),
$$

where we are using (13) and the fact that $\operatorname{Cov}\left(m\left(a^{\prime}\right), m(a)\right)=\operatorname{Var}\left(m\left(a^{\prime}\right)\right)$. The agent can then ensure himself the same expected outcome at a strictly lower variance by reducing $\Delta^{\prime}$ and increasing $\Delta$ appropriately. This proves that the agent will never take two unknown actions to the left of $h_{t}$.

We already observed in the text that if the agent does put some income into an unknown action, he will never put in more than $\underline{\theta}$. In any period $t$, the agent will therefore put all his income into the best known action or he will put a fraction $(1-\underline{\theta})$ into the best known action and the rest into an unknown action to the right of $h_{t}$. Consider first the constrained problem in which the agent 
has to experiment to the right of $h_{t}$, which is given by

$$
\max _{\Delta_{t} \in[0, \infty)} \mathrm{E}\left[u\left((1-\underline{\theta}) m\left(\bar{a}_{t}\right)+\underline{\theta} m\left(h_{t}\right)+\underline{\theta} \mu \Delta_{t}+\underline{\theta} \sqrt{\Delta_{t}} \sigma z_{t}\right)\right] .
$$

This is exactly the same problem as the constrained problem in the main model if the drift and the variance of the Brownian motion were given by $\underline{\theta} \mu$ and $\underline{\theta}^{2} \sigma^{2}$ and the outcome generated by the right-most action were $(1-\underline{\theta}) m\left(\bar{a}_{t}\right)+\underline{\theta} m\left(h_{t}\right)$. Moreover, the agent's unconstrained problemwhich involves comparing the agent's expected utility from taking the constrained optimal action with his utility from taking the best action-is also the same as appropriately specified version of the main model. Except for the comparative statics with respect to $\bar{m}_{t}$ and $\underline{\theta}$, all the claims in the proposition therefore follow from Propositions 1-3.

For the comparative static with respect to $\underline{\theta}$, suppose that the $\Delta_{t}$ that maximizes the agent's constrained problem is strictly positive and denote it by $\Delta^{*}$. If the agent does experiment, his expected outcome is then given by $\mathrm{E}\left[m_{t}\right]=m\left(h_{t}\right)+\mu \underline{\theta} \Delta^{*}$ and the variance is given by $\operatorname{Var}\left(m_{t}\right)=$ $\sigma^{2} \underline{\theta}^{2} \Delta^{*}$. Suppose now that the minimum feasible scale $\underline{\theta}$ is reduced to $\underline{\underline{\theta}}<\underline{\theta}$. The agent can then achieve the same expected outcome with a lower variance by reducing the fraction of income he invests in the unknown action to $\underline{\underline{\theta}}$ and increasing the step size. This has two implications. First, since expected utility is concave in the step size, the new optimal step size is strictly larger than $\Delta^{*}$. This proves that reduction in $\underline{\theta}$ increases the optimal step size. Second, the above observation implies that a reduction in $\underline{\theta}$ increases the agent's expected utility from taking the optimal action. It then follows from the proof of Proposition 3 that a reduction in $\underline{\theta}$ reduces $\widetilde{m}\left(\bar{m}_{t}\right)$.

Finally, consider the comparative statics with respect to $\bar{m}_{t}$. The result that the optimal step size $\Delta\left(m\left(h_{t}\right)\right)$ is increasing in $\bar{m}_{t}$ follows immediately from the fact that in the main model the optimal step size is increasing in $m\left(h_{t}\right)$. To see that the threshold $\widetilde{m}\left(\bar{m}_{t}\right)$ can now be decreasing in $\bar{m}_{t}$, suppose that the utility function is given by (10). It then follows from Section 4.4 that

$$
\widetilde{m}\left(\bar{m}_{t-1}\right)=\frac{1}{\underline{\theta}} \widehat{m}-\frac{(1-\underline{\theta})}{\underline{\theta}} \bar{m}_{t-1}+\frac{\left(\beta-\frac{2 \mu}{\underline{\theta} \sigma^{2}}\right)}{\underline{\theta} \alpha \beta}\left(u\left(\bar{m}_{t-1}\right)-u(\widehat{m})\right) .
$$

Differentiating this expression we get

$$
\frac{\mathrm{d} \widetilde{m}\left(\bar{m}_{t-1}\right)}{\mathrm{d} \bar{m}_{t-1}}=-\frac{(1-\underline{\theta})}{\underline{\theta}}+\frac{\left(\beta-\frac{2 \mu}{\underline{\theta} \sigma^{2}}\right)}{\underline{\theta} \alpha \beta}(\alpha+\beta \exp (-\beta \bar{m})) .
$$

This expression will be negative if

$$
\left(\beta-\frac{2 \mu}{\underline{\theta} \sigma^{2}}\right) \beta \exp (-\beta \bar{m})<\alpha\left(\frac{2 \mu}{\underline{\theta} \sigma^{2}}-\beta \underline{\theta}\right)
$$


which, in turn, will be the case if

$$
\frac{2 \mu}{\underline{\theta}^{2} \sigma^{2}}>\beta>\frac{2 \mu}{\underline{\theta} \sigma^{2}}
$$

and $\bar{m}$ is sufficiently large.

\section{Stochastic Processes}

In this section we analyze the case in which the underlying environment is the realized path of a geometric Brownian motion. Recall that the agent's problem is

$$
\max _{\Delta} E[u(m(\Delta))]
$$

where $m(\Delta)$ is now given by

$$
m(\Delta)=m_{0} \exp (\mu \Delta+\sigma W(\Delta))
$$

and $W(\Delta)$ is a standard Brownian motion.

Now let $z$ denote a random variable with a standard lognormal distribution. We can then write

$$
m(\Delta)=M(\Delta)+\sqrt{\frac{V(\Delta)}{\operatorname{Var}(z)}}(z-E[z]),
$$

which allows us to rewrite the problem as

$$
\max _{\Delta} E\left[u\left(M(\Delta)+\sqrt{\frac{V(\Delta)}{\operatorname{Var}(z)}}(z-E[z])\right)\right]
$$

The first derivative is

$$
\frac{d E[u(\ldots)]}{d \Delta}=M^{\prime}(\Delta) E\left[u^{\prime}(. .)\right]+\frac{1}{2} \sqrt{\frac{\operatorname{Var}(z)}{V(\Delta)}} V^{\prime}(\Delta) E\left[u^{\prime}(\ldots)(z-E[z])\right] .
$$

We wish to examine the derivative at $\Delta=0$. For this purpose, rewrite the derivative as

$$
\frac{d E[u(\ldots)]}{d \Delta}=M^{\prime}(\Delta) E\left[u^{\prime}(. .)\right]+\frac{1}{2} \sqrt{\operatorname{Var}(z)} V^{\prime}(\Delta)\left[\frac{E\left[u^{\prime}(\ldots)(z-E[z])\right]}{\sqrt{V(\Delta)}}\right] .
$$

At $\Delta=0$, both the numerator and the denominator of the term in squared brackets are zero. Applying l'Hopital to the second term on the RHS we get

$$
\begin{aligned}
& \sqrt{V(\Delta)} \sqrt{\operatorname{Var}(z)} \frac{V^{\prime \prime}(\Delta)}{V^{\prime}(\Delta)} E\left[u^{\prime}(\ldots)(z-E[z])\right] \\
& +\sqrt{\operatorname{Var}(z)}\left(\sqrt{V(\Delta)} M^{\prime}(\Delta) E\left[u^{\prime \prime}(\ldots)(z-E[z])\right]+\frac{1}{2} \sqrt{\operatorname{Var}(z)} V^{\prime}(\Delta) E\left[u^{\prime \prime}(\ldots)(z-E[z])^{2}\right]\right)
\end{aligned}
$$


At $\Delta=0$ this becomes

$$
\operatorname{Var}(z)^{2} \frac{1}{2} V^{\prime}(0) u^{\prime \prime}\left(m_{0}\right)
$$

So at $\Delta=0$ we have

$$
\begin{aligned}
\frac{d E[u(\ldots)]}{d \Delta} & =M^{\prime}(0) u^{\prime}\left(m_{0}\right)+\operatorname{Var}(z)^{2} \frac{1}{2} V^{\prime}(0) u^{\prime \prime}\left(m_{0}\right) \\
& =\frac{1}{2} V^{\prime}(0) u^{\prime}\left(m_{0}\right)\left[\frac{2 M^{\prime}(0)}{V^{\prime}(0)}-\left(-\frac{u^{\prime \prime}\left(m_{0}\right)}{u^{\prime}\left(m_{0}\right)}\right) \operatorname{Var}(z)^{2}\right]
\end{aligned}
$$

Note that this expression is exactly the same as for the Brownian motion in which case $z$ is distributed normally and $\operatorname{Var}(z)=1$.

From the properties of the geometric Brownian motion we know that

$$
M^{\prime}(0)=m_{0}\left(\mu+\frac{1}{2} \sigma^{2}\right)
$$

and

$$
V^{\prime}(0)=m_{0}^{2} \sigma^{2}
$$

And so we have

$$
\begin{aligned}
\frac{d E[u(\ldots)]}{d \Delta} & =M^{\prime}(0) u^{\prime}\left(m_{0}\right)+\operatorname{Var}(z)^{2} \frac{1}{2} V^{\prime}(0) u^{\prime \prime}\left(m_{0}\right) \\
& =\frac{1}{2} V^{\prime}(0) u^{\prime}\left(m_{0}\right)\left[\frac{2\left(\mu+\frac{1}{2} \sigma^{2}\right)}{m_{0} \sigma^{2}}-\left(-\frac{u^{\prime \prime}\left(m_{0}\right)}{u^{\prime}\left(m_{0}\right)}\right) \operatorname{Var}(z)^{2}\right]
\end{aligned}
$$

The key difference to the Brownian motion case is that the risk adjusted return is now decreasing in $m_{0}$ (the first expression in the brackets). The richer you are, the worse therefore the opportunity for innovation. The sign of the derivative, however, depends on how this compares to declining absolute risk aversion, given by the second term in the brackets. The technological opportunities for innovation would dominate if the coefficient of absolute risk aversion were constant, that is, if we had a regular exponential utility function $u(m)=-\exp (-\beta m)$. In this case, the above becomes

$$
\frac{d E[u(\ldots)]}{d \Delta}=\frac{1}{2} V^{\prime}(0) u^{\prime}\left(m_{0}\right)\left[\frac{2\left(\mu+\frac{1}{2} \sigma^{2}\right)}{m_{0} \sigma^{2}}-\beta \operatorname{Var}(z)^{2}\right] .
$$

and a threshold value of $m_{0}$ exists such that the first derivative is negative at $\Delta=0$ for starting performance above this threshold.

To capture both declining innovation opportunities and declining risk aversion, apply instead the linex utility function used in Section 4.4 Setting $u(m)=a m-\exp (-\beta m)$, the above becomes

$$
\frac{d E[u(\ldots)]}{d \Delta}=\frac{1}{2} V^{\prime}(0) u^{\prime}\left(m_{0}\right)\left[\frac{2\left(\mu+\frac{1}{2} \sigma^{2}\right)}{m_{0} \sigma^{2}}-\left(\frac{\beta^{2} \exp \left(-\beta m_{0}\right)}{a+\beta \exp \left(-\beta m_{0}\right)}\right) \operatorname{Var}(z)^{2}\right]
$$


or

$$
\frac{d E[u(\ldots)]}{d \Delta}=\frac{1}{2} V^{\prime}(0) u^{\prime}\left(m_{0}\right) m_{0} \operatorname{Var}(z)^{2}\left[\frac{2\left(\mu+\frac{1}{2} \sigma^{2}\right)}{\sigma^{2} \operatorname{Var}(z)^{2}}-\left(m_{0} \frac{\beta^{2} \exp \left(-\beta m_{0}\right)}{a+\beta \exp \left(-\beta m_{0}\right)}\right)\right]
$$

Numerical calculations show that the second expression in the brackets is single peaked, reaching a maximum for moderate levels of performance. Thus, if the first expression is large - the innovation opportunities are good - then all agents have a marginal incentive to experiment. For less appealing technological opportunities (lower drift or higher variance of the stochastic process), it will be the moderate performers who first experience a disincentive for initial experimentation.

How agents trade-off the attractiveness of innovation opportunities and risk varies, therefore, in the agent's tolerance for risk. Take the following utility function that is the sum of two standard utility functions that are frequently used when outcomes are lognormally distributed.

$$
\begin{aligned}
u(m) & =a \log m-\frac{1}{m^{b}} \\
& =a \log m-\exp (-b \log m)
\end{aligned}
$$

Straightforward calculations show that the coefficient of risk aversion and prudence are given by

$$
\begin{aligned}
& r=\frac{b+a m^{b}+b^{2}}{m\left(b+a m^{b}\right)} \\
& p=\frac{2 b+2 a m^{b}+3 b^{2}+b^{3}}{m\left(b+a m^{b}+b^{2}\right)}
\end{aligned}
$$

As both are decreasing, and DARA and DAP imply standardness, this utility function satisfies standard risk aversion and the requirements of our model.

Looking again at the first derivative at $\Delta=0$,

$$
\begin{aligned}
\frac{d E[u(\ldots)]}{d \Delta} & =\frac{1}{2} V^{\prime}(0) u^{\prime}\left(m_{0}\right)\left[\frac{2\left(\mu+\frac{1}{2} \sigma^{2}\right)}{m_{0} \sigma^{2}}-\left(\frac{b+a m_{0}^{b}+b^{2}}{m_{0}\left(b+a m_{0}^{b}\right)}\right) \operatorname{Var}(z)^{2}\right] \\
& =\frac{1}{2 m_{0}} V^{\prime}(0) u^{\prime}\left(m_{0}\right)\left[\frac{2\left(\mu+\frac{1}{2} \sigma^{2}\right)}{\sigma^{2}}-\left(\frac{b+a m_{0}^{b}+b^{2}}{\left(b+a m_{0}^{b}\right)}\right) \operatorname{Var}(z)^{2}\right] .
\end{aligned}
$$

which reveals that, for this utility function, the declining innovation opportunities of the geometric Brownian motion are dominated by declining risk aversion. Thus, within our model, there exists preferences such that the comparative static remains, even with a geometric Brownian motion, that if any agents do not have the incentive to experiment, it is the lowest performing agents only. This, unfortunately, is not a complete analysis of behavior, and we must leave that task for future work. 